\title{
Feeding Potential of Fruit Fly (Diptera: Tephritidae) on Different Artificial Diets under Laboratory Conditions
}

\section{Iqra Aslam ${ }^{1}$, Muhammad Ahsan Khan², Taskeen Ahmad ${ }^{2 *}$, Tayybah Gul ${ }^{2}$, Namrah Nazeer ${ }^{1}$, Farwa Aslam ${ }^{1}$ and Kiran Shehzadi ${ }^{1}$}

${ }^{1}$ Department of Zoology, Wildlife and Fisheries, University of Agriculture Faisalabad, Pakistan

${ }^{2}$ Department of Entomology, University of Agriculture Faisalabad, Pakistan

*Corresponding Author: Taskeen Ahmad, Department of Entomology, University of Agriculture Faisalabad, Pakistan.

Received: September 26, 2019; Published: October 22, 2019

DOI: 10.31080/ASAG.2019.03.0693

\begin{abstract}
Fruit flies are a serious pest of fruits and vegetables. They can lose to the GDP of any country by damaging the fruit and vegetable industry. It has a great economic threat to worldwide. In the present study, five different artificial diets were used and the life cycle (Fecundity, egg hatchability, larval development, pupal formation, adult emergence, and sex ratio) of Bactrocera zonata was checked. Results showed that diet V (100ml water, $18.8 \mathrm{~g}$ sugar, 0.07 sodium benzoate, and $5 \mathrm{~g}$ beaker yeast) gives significant results in all life stages of the fruit fly. Fecundity, egg hatchability, larval development, pupal formation, adult emergence, and the sex ratio was observed maximum in the diet $\mathrm{V}$ and diet $\mathrm{I}(100 \mathrm{ml}$ water, $18.8 \mathrm{~g}$ sugar, and 0.07 sodium benzoate) gives minimum means results of the life cycle of $B$. zonata. The results of this study showed that all types of diets had a significant effect on the B. zonata life cycle but diet 5 showed more significant $(\mathrm{p}<0.05)$ results than other artificial diets.
\end{abstract}

Keywords: Bactrocera zonata; Fruit Fly; Diptera; Tephritidae

\section{Introduction}

The most serious fruit fly pests of the specialized sector having approx. 4000 species, are belonging to family Tephritidae and leads to premature ripening and damaging of fruits by feeding them. Up to $99 \%$ of the result may be broken by way of fruit fly while left out of control. As such, the pleasant and industrial cost of the crop that affects the fruit yield and reduces the fruit and their infestation no longer [1]. In Pakistan, the essential and different fruit flies that damage the veggies, Bactrocera zonata (saunders); damage the peach, eastern fruit fly, Bactrocera dorsalis Hendel; melon fruit fly, Bactrocera cucurbitae and Bactrocera ciliates Loew [2]. Annually, these fruit flies cause losses of veggies up to climactic levels with an anticipated value of 200 dollars. A yield of guava plants with the aid of small farmers go through $25-70 \%$ losses attributable to the inclined types is measured [3].
The prevalence of fruit flies at the same time influences quantity and high quality of culmination. The fruit fly maggots bore in the fruit for feeding of pulp which is dangerous for human use. The result [4] is the rotting starts that evolved at the point of perforation. In the host plant species, a great loss to a higher level is caused by fruit flies. According to the whole study of science, the most hazardous species of pest is the eastern fruit fly that motives a five-100 percentage loss to numerous culmination [5]. Bactrocera zonata laid 22 eggs per day in a line and approximately 500 to 800 eggs throughout their life cycle. The female gender of fruit flies injects their eggs to the pores and outer covering of ripening culmination. In hot climatic conditions, eggs burst out in 5 to 3 days to gives rise to their off springs. The hatching ability of eggs is appreciably improved with the aid of decreased temperature [6]. When larvae hatching starts consuming, and make damage at first, 
however, it may continue to be near collectively in feeding until then they completely have grown. The development of larva completes within 6-15 days having favorable temperature [7]. The fruit fly larvae damage the fruit and ideally pupate within the soil [8]. In the course in hot weather early with in the morning, the adult emerges in the largest number and throughout the cool climate emerges extra irregularly. Most of the adults emerge in morning time 8 to 10 [9]. Fruit flies are very energetic and active early in the morning and their interest decrease steadily toward mid of day with upward thrust in relative humidity, temperature, and fall [10]. This study aimed to observe the life cycle of B. zonata by feeding on the different artificial diets. The main purpose of this study was to check the development of B. zonata on an artificial diet and compared it with the previous studies.

\section{Materials and Methods}

The experiments were managed through the summer at the laboratory of the Entomology department in Agriculture University Faisalabad to check the economical natural and unnatural larval meal by the rearing of B. zonata under controlled laboratory circumstances. In proper concentration the ingredient was used for the artificial diet is egg, sugar, baker, yeast, banana, sodium benzoate, water, protein hydrolysate. The different types of parameters were studied, in this experiment include the percentage of eggs hatchability, development of larvae into pupae, adults' emergence rate from pupae, the fecundity of female and sex ratio, life table and survivorship. The liquid medium diets have measured the percentage of eggs. The second experiment's solid diets treatments were measured the other parameters that were applied.

\section{Insect Collection, culture and rearing}

The insect pair of fruit fly male and female was collected from the 9 square fields in the University of Agriculture Faisalabad. The special type of traps was used for capturing the fruit fly, pheromone was used to capture both male and female. Within one hour the flies was capture from the field then transfer carefully in laboratory condition for rearing the F1 generation. The collection of fruit fly from rearing cages with the help of a collection instrument at the department of Entomology laboratory. For insect collection different methods are used, the plastic bottle collection instruments and damage fruits having $1 \mathrm{~mm}$ holes throughout. The fruit flies laying eggs in rearing cages for eggs laying having stain inside with the guava diluted juice. After make sure (1-2) eggs presence the collection instrument was wash the small amount of tap wa- ter. The rearing room conditions were $23 \mathrm{C} \pm 20 \mathrm{C}$ temperature and humidity is $60-70 \%$ with a photoperiod. Moreover, after two days changing the diet slide and cleaning of rearing cages was necessary for avoid the fungus development and contamination. The pupae were collected from the infected fruits guava that was sited inside the plastic cages $(25 \times 25 \times 30)$. Cages from the side were made of mesh for ventilation. Adult flies were emerged then provided after two days with the D1 mixture of "100 ml water, sugar $18.8 \mathrm{~g}$, sodium benzoate $0.07 \mathrm{~g}$. Firstly tissue dip in the diet and placed in the Petri plate then this Petri plate placed in cages for feed. The inside top of the cage diet is spread with the help of tissue that was a dip in the liquid diet. The cucumber was placed in the cage for the laid egg. After the egg was laid then cucumber was transferred into another cage over the tray of sand.

\section{Liquid diets experiment}

The percent hatchability of eggs was studied in this experimental study by various liquid diet or treatment. The number of different components used for the preparation of these feeds. $100 \mathrm{ml}$ distilled water was used in each diet. To prepare a homogenous solution, the measured quantity of each ingredient was added in each beaker. Replicate thrice each treatment. The beaker was placed in an incubator at $25 \pm 3^{\circ} \mathrm{C}, 70-75 \%$ relative humidity for 3-4 days.

\section{Larvae rearing}

Collected eggs were put on the semi-artificial diet inside the plastic tray to prevent desiccation. Containers cover were aerated with 1-3 mm diameter holes during larval duration period. On metal shelves, the larval trays were stored. Larval trays were put in aerated cages provided with wet sterilized sand on the bottom before the maturation of larvae. Mature larvae begin to leave the diet then jump to the sand for pupation after one week.

\section{Handling pupae}

After four days of pupation, the pupae were collected. From the sand, pupae were strained and kept in the adult rearing cages until emergence. Pupae were calculated based on the original number of eggs that were put into the diet and also determine the number of pupae was recovered. Five different artificial diets were given to the fruit fly from egg hatching to adult.

\section{Artificial Diet}

- $\quad$ Diet I: $100 \mathrm{ml}$ water, $18.8 \mathrm{~g}$ sugar and 0.07 sodium benzoate.

- Diet II: $100 \mathrm{ml}$ water, $18.8 \mathrm{~g}$ sugar, 0.07 sodium benzoate, and $5 g$ yeast. 
- $\quad$ Diet III: $100 \mathrm{ml}$ water, $18.8 \mathrm{~g}$ sugar, 0.07 sodium benzoate, $5 \mathrm{~g}$ protein hydrolysate, and beaker yeast.

- Diet IV: $100 \mathrm{ml}$ water, $18.8 \mathrm{~g}$ sugar, 0.07 sodium benzoate, $5 \mathrm{~g}$ protein hydrolysate.

- Diet V: $100 \mathrm{ml}$ water, $18.8 \mathrm{~g}$ sugar, 0.07 sodium benzoate, and $5 \mathrm{~g}$ beaker yeast.

\section{Preparation of diet}

In a plastic tub, a known quantity of sugar was mixed with a known quantity of distilled water. Various other solid ingredients such as sodium benzoate, yeast, protein hydrolysate, baker's yeast along with water were added after sugar dilution.

\section{Fecundity}

Fresh fruit is collected from the garden that was placed into all cages. All cages have a different diet for fruit fly. Fresh fruit was placed into the all adult rearing cages that containing the 55 pairs of 7 days old flies of B. zonata for depositing the egg in it. Fresh cucumber is also a good medium for egg-laying of the fruit fly. An artificial diet is provided to all 5 cages with the help of tissue. Fluorescent lamps were closed and cages were placed on the shelve to avoid any contamination of diet. Estimation condition is adjusted on the $30 \pm 3^{\circ} \mathrm{C} 60-70 \%$ relative humidity and $15: 10$ photoperiod.

\section{Pupae formation}

The known number of eggs was put in a tray; containing larval diet. These trays were then shifted to another box having sand as a source medium for the emergence of pupae and their subsequent collection. The pupae formed during the experiment were sieved by using mesh-10 sieve. Then the pupa formation rate was recorded and collected.

\section{Emergence of adult}

The collected pupae were shifted to a rearing cage of adult $(2 \times$ $1 \times 1.45$ ) cubic meter which contains yeast, water, sodium benzoate, and protein hydrolysate as an adult diet in the Petri dishes. Maintenance of moisture rate and supply of water was made continuous in the adult rearing cages, in a small jar, cotton was soaked in the distilled water to clean it. By visual observation, the number of adults emerges was calculated. On a daily basis, the percentage of sex ratio of adult emergence from pupae was determined until all adult has emerged.

\section{Data collection}

Food, as baits were given with different concentrations of artificial diet and food, provide fruit as choice and free choice to the fruit fly. The experiment was run for 1 month and after an appropriate time, data was collected. At each interval of time, different morphological changes were noted. Moderate temperature and humidity and photoperiod were required to experiment.

Data analysis

All the five experiments, each with three replicates were analyzed by Complete Randomized Design (CRD) and one way ANOVA test and means were separated by Tuckey's HSD (All-Pairwise Comparison) Test at $5 \%$ level of Significance $(\mathrm{p}<0.05)$.

\section{Results}

\section{Fecundity and egg hatchability rate}

Results of fecundity and egg hatchability showed that all the treatment have a significant effect but diet 5 give maximum significance. Fecundity and egg hatchability rate of diet $5(100 \mathrm{ml}$ water, 18.8g sugar, 0.07 sodium benzoate and $5 \mathrm{~g}$ beaker yeast) was 99 and $98 \%$ followed by the diet $4(100 \mathrm{ml}$ water, $18.8 \mathrm{~g}$ sugar, 0.07 sodium benzoate, 5 g protein hydrolysate) 97 and 94\%, diet $3(100 \mathrm{ml}$ water, $18.8 \mathrm{~g}$ sugar, 0.07 sodium benzoate, $5 \mathrm{~g}$ protein hydrolysate and beaker yeast) 95 and $92 \%$ diet $2(100 \mathrm{ml}$ water, 18.8g sugar, 0.07 sodium benzoate and $5 \mathrm{~g}$ yeast) 92 and $90 \%$ and diet $1(100 \mathrm{ml}$ water, $18.8 \mathrm{~g}$ sugar and 0.07 sodium benzoate) gives the minimum fecundity rate 90 and $88 \%$ respectively.

\section{Larval and pupal development}

Results of larval and pupal development on the artificial diet were significantly different from each other and larval and pupal development were also observed maximum in diet 5. Larval and pupal development on diet 5 was 96 and 93\% and minimum in diet 187 and 82\%. Other artificial diets (4, 3 and 2) have 94 and 90, 92 and 87 and 90 and $84 \%$ respectively.

\section{Adult emergence and sex ratio}

Results of adult emergence and sex ratio were significantly different from each other. Adult emergence and sex ratio were also observed in diet 5 and minimum observed in diet 1 . Results of sex ratio showed that female's adults were observed maximum as compared to the females. Results of adult emergence on artificial diet were 90, 85, 82, 79 and $75 \%$ in all diets (5, 4, 3, 2 and 1). Sex ratio results showed that 76 females and 38 males were observed in the diet 5 followed by 69 females and 34 males in diet 4, 65 females and 32 males in diet 3, 59 females and 29 males in diet 2 and 53 females and 26 males was observed in diet 1 respectively. 
Feeding Potential of Fruit Fly (Diptera: Tephritidae) on Different Artificial Diets under Laboratory Conditions

\begin{tabular}{|l|c|c|c|c|c|c|c|c|}
\hline $\begin{array}{l}\text { S r. } \\
\text { No. }\end{array}$ & Treatments & $\begin{array}{c}\text { Fecundity } \\
\pm \text { SE }\end{array}$ & $\begin{array}{c}\text { Egg Hatch- } \\
\text { ability } \pm \text { SE }\end{array}$ & $\begin{array}{c}\text { larval develop- } \\
\text { ment } \pm \text { SE }\end{array}$ & $\begin{array}{c}\text { Pupal for- } \\
\text { mation } \pm \text { SE }\end{array}$ & $\begin{array}{c}\text { Adult emer- } \\
\text { gence } \pm \text { SE }\end{array}$ & Male \pm SE & Female \pm SE \\
\hline 1 & Diet 1 & $90 \pm 0.8563$ & $88 \pm 0.5774$ & $87 \pm 0.8165$ & $82 \pm 0.8065$ & $75 \pm 0.8065$ & $26 \pm 0.7601$ & $53 \pm 0.6992$ \\
\hline 2 & Diet 2 & $92 \pm 0.8563$ & $90 \pm 0.5774$ & $90 \pm 0.8165$ & $84 \pm 0.8065$ & $79 \pm 0.8065$ & $29 \pm 0.7601$ & $59 \pm 0.6992$ \\
\hline 3 & Diet 3 & $95 \pm 0.8563$ & $92 \pm 0.5774$ & $92 \pm 0.8165$ & $87 \pm 0.8065$ & $82 \pm 0.8065$ & $32 \pm 0.7601$ & $65 \pm 0.6992$ \\
\hline 4 & Diet 4 & $97 \pm 0.8563$ & $94 \pm 0.5774$ & $94 \pm 0.8165$ & $90 \pm 0.8065$ & $85 \pm 0.8065$ & $34 \pm 0.7601$ & $69 \pm 0.6992$ \\
\hline 5 & Diet 5 & $99 \pm 0.8563$ & $98 \pm 0.5774$ & $96 \pm 0.8165$ & $93 \pm 0.8065$ & $90 \pm 0.8065$ & $38 \pm 0.7601$ & $76 \pm 0.6992$ \\
\hline
\end{tabular}

Table: Fecundity, egg hatchability, larval development, pupal formation, adult emergence and sex ratio of B. zonata in artificial diets.

\section{Discussion}

The performance of the artificial diet was analyzed based on their effectiveness on the life cycle of B. zonata. Results showed that among all artificial diets, D5 $(100 \mathrm{ml}$ water, $17.7 \mathrm{~g}$ sugar, 0.06 sodium benzoate and $5.2 \mathrm{~g}$ beaker yeast) was significant to enhance the egg hatching ability 98\%, larval 96\%, pupal formation 93\% and adult emergence $90 \%$ also increased and considerable female and the male ratio was achieved. The findings of the current study are similar to the study of Shinwari., et al. [8] who used above mentioned artificial diets against fruit fly for the evaluation of the growth rate of the fruit fly, they checked these diets at different doses against fruit fly for its all stages. It reveals that doses of sodium benzoate and beaker yeast show the most effective results on the growth of fruit fly in egg hatchability $86 \%$ and larvae and pupae formation is $80 \%, 78 \%$ respectively. Ragab [11] performed a study to evaluate diet preference as food to a fruit fly. They used different artificial diets and their results showed that a mixture of artificial and natural diets was most preferable as a portion of food and results are similar to our study that shows effective development in the life stages of fruit fly under no choice experimental test. Sookar., et al. [12] studied that water was also effective in the case of fecundity and flies' fertility and water had similar effectiveness like tested juices. The total egg production was $90 \%$ during the first three weeks and these eggs were collected from adult ovipositional cages which carrying fifty thousand flies. The larval diet contain ground maize, wheat bran, benzoic acid, waste brewer's yeast, sugarcane bagasse, sugarcane sugar, nipagin, and water resulted in the following values for the quality of control parameters such as $85 \%$ of egg hatching, $67 \%$ recovery of pupa, pupal formation was $89 \%$ and $65 \%$ adult emerged and grow into flies.

\section{Conclusion}

The present study was performed to find out the effect of several types of food on fruit fly Bactrocera zonata and Bactrocera cu- curbitae growth and also investigated that either artificial food or diets improve the growth. For this purpose, various types of artificial and natural diets were used to check their effects on egg hatching ability, larval formation, pupal formation, adult emergence, and sex ratio, and data was noted after two days. Results showed that among all diets D5 was significant to enhance the egg hatching ability, larval and pupal formation, adult emergence also increased and a considerable female ratio was achieved. Results showed that among all artificial diets, D5 was significant to enhance the egg hatching ability 98\%, larval 96\%, pupal formation 93\% and adult emergence $90 \%$ also increased and considerable female and the male ratio was achieved.

\section{Bibliography}

1. Hardy D. "Taxonomy and distribution of the Oriental fruit fly and related species Tephritidae-Diptera”. 45 (1997): 56-58.

2. John MS., et al. "Economic losses totephritid fruit flies (Dipteraephritidae)”. Pakistan Crop Protection 17 (1997):159-164.

3. Khattak SUK., et al. "Integrated management of fruit flies. Manual of Integrated Pest Management on Fruit fly and Termites". Director General Agricultural Extension NWFP (2006): 12-20.

4. Hull CD and BW Cribb. "Ultrastructure of the antennal sensilla of Queensland fruit fly, Bactrocera tryoni (Froggatt) (Diptera: Tephritidae)". International Journal of Insect Morphology and Embryology 26 (1997): 27-34.

5. Placido-Silva., et al. "Effects of different protein concentrations on longevity and feeding behavior of two adult populations of (Ceratitiscapitata) Wiedemann Diptera: Tephritidae". Neotropical Entomology 35(6): 747-752.

6. Kapoor VC and ML Agarwal. "Fruit flies and their natural enemies in India". In Fruit flies of economic importance. Proceed. CEC/IOBC Int. Sym. Athens Greece (1983): 104-105. 
7. Shehata N F., et al. "Biological studies on the peach fruit fly, Bactrocera zonata (Saunders) in Egypt". Journal of Applied Sciences Research 4 (2008): 1103-1106.

8. Shinwari I., et al. "Evaluation of artificial larval diets for rearing of fruit fly Bactrocera zonataDiptera: Tephritidae under laboratory condition". Journal of Entomology and Zoology Studies 3 (2015): 189-193.

9. Schroeder WJ., et al. "Protein products for rearing three species of larval Tephritidae". Journal of Economic Entomology 65 (2000): 969-972.

10. Alamzeb AUK., et al. "Control of fruit flies using neem extract. Manual of Integrated Pest Management on Fruit fly and Termites". Directorate General Agricultural extension 6 (2006): 65-69.

11. Rabab RA., et al. "Laboratory rearing of the peach fruit fly Bactrocera zonata (Saunders) (Diptera: Tephritidae) on semiartificial diet based on soybean protein". Alexandria Journal of Agricultural Sciences 61 (2016): 24-36.

12. Sookar P., et al. "Artificial rearing of the peach fruit fly Bactrocera zonata (Diptera: Tephritidae)". International Journal of Tropical Insect Science 34 (2014): 99-107.

\section{Volume 3 Issue 11 November 2019}

(C) All rights are reserved by Taskeen Ahmad., et al. 